\title{
“梯度脸”局部高阶主方向模式的人脸识别
}

\author{
叶学义, 王涛, 应娜, 钱丁炜 \\ (杭州电子科技大学通信工程学院 杭州 310018) \\ (2990177234@qq.com)
}

\begin{abstract}
摘 要：现有高阶特征的人脸识别由于对噪声敏感导致鲁棒性差以及特征冗长，为此提出一种基于“梯度脸”的局部 高阶主方向模式的人脸识别方法. 首先设计梯度脸卷积算子，计算像素的多方向梯度分量和，以构造梯度脸；然后 在梯度脸上引人主方向分组策略表征其高阶导数特征，以局部邻域高阶导数方向变化的特征码形成主方向特征图; 最后分块统计其直方图特征并级联，并利用多分类支持向量机完成分类识别. 在多个公开人脸库中的实验结果表明， 该方法对光照、表情和面部遮挡等变化因素具有良好的鲁棒性，以及更高的识别效率.
\end{abstract}

关键词: 人脸识别; 局部导数模式; 梯度脸; 主方向分组策略

中图法分类号: TP391.41ＤOI: 10.3724/SP.J.1089.2021.18789

\section{Face Recognition with Local High-Order Principal Direction Pattern Based on "Gradient Face"}

\author{
Ye Xueyi, Wang Tao*, Ying Na, and Qian Dingwei \\ (School of Communication Engineering, Hangzhou Dianzi University, Hangzhou 310018)
}

\begin{abstract}
Pointing to weak robustness caused by the noise sensitivity and feature redundancy of present face recognition methods with high-order features, a new method of the local high-order principal direction pattern based on "gradient face" is proposed. Firstly, the gradient face convolution operator designed is used to compute the sum of multi-directional gradient components of pixels to construct a gradient face. Then, the principal direction grouping strategy is introduced on the gradient face to characterize its high-order derivative features, and a principal direction feature map is formed according to the feature code of high-order derivatives direction changes in local neighborhood. Finally, block statistics and cascading of histogram features are made a vector to be input in to a support vector machine for multi-classification. Experimental results of several public face databases show that the proposed method is robust to changes in illumination, expression, and facial occlusion and has higher recognition efficiency.
\end{abstract}

Key words: face recognition; local derivative pattern; gradient face; principal direction grouping strategy

人脸识别作为普遍为人熟知但又颇具挑战性 的课题, 还存在许多需要解决的问题, 如光照、表 情、遮挡和姿态等变化因素以及噪声干扰对人脸识
别的准确率影响较大, 在一定条件下会影响原有方 法的识别效果. 同时, 人们对人脸识别的实时性要 求也使识别效率成为另一个值得关注的指标.

收稿日期: 2020-11-21; 修回日期: 2021-03-09. 基金项目：国家自然科学基金(60802047，60702018). 叶学义(1973一), 男，博士， 副教授, 硕士生导师, 主要研究方向为模式识别、信息安全; 王涛(1990一), 男, 硕士研究生, 论文通讯作者, 主要研究方向为人脸识 别; 应娜(1978一), 女, 博士, 副教授, 硕士生导师, 主要研究方向为信号处理; 钱丁炜(1996-), 男, 硕士研究生, 主要研究方向为 人脸识别. 
人脸识别大多数研究中都使用了纹理分析和 分类. 纹理反映了图像的视觉特征, 表征一个物体 或其局部的表面特性 ${ }^{[1]}$, 其被广泛地用作人脸特征 描述符，以区分和检索图像 ${ }^{[2-3]}$. 全局纹理描述符 可以刻画人脸的粗略信息, 具有代表性的方法有 主成分分析法 (principal component analysis, PCA) ${ }^{[4]}$ 和线性判别分析法 (linear discriminant analysis, LDA $)^{[5]}$ 等. 而局部纹理描述符更能反映 人脸图像鉴别信息的细节部分, 通常它对光照和 表情等变化因素具有较强的鲁棒性. 典型的代表方 法有方向梯度直方图(histogram of oriented gradient, $\mathrm{HOG})^{[6]}$ 、Gabor 变换 ${ }^{[7]}$ 、局部三值模式(local ternary

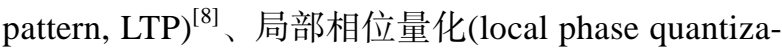
tion, LPQ $)^{[9]}$ 和局部二值模式(local binary pattern, LBP $)^{[10]}$. 在这些方法中, LBP 及其衍生方法 ${ }^{[11-16]}$ 因 其具有高性能和计算复杂度低的特点, 近些年受到 了广泛的关注.

Ojala 等 ${ }^{[10]}$ 提出的 LBP 是一种著名的且被广泛 研究应用的局部纹理描述符. LBP 本质上是图像的 一阶导数模式, 是一种由二值导数方向组合而成 的微模式. 但是一阶导数模式提取的特征图往往 会产生较粗的边缘, 轮廓不够清晰, 对图像细节的 表征能力不够 ${ }^{[17-18]}$. 高阶导数对图像精细细节(如 细线、边缘等灰度变化剧烈区域)的增强作用要比 一阶导数好得多, 但是其对孤立像素点的响应也 更为强烈, 容易放大噪声, 这也是其弊端. 许多学 者对图像的高阶导数展开了深人的研究, 提出了 许多优秀的高阶导数方法 ${ }^{[14-16]}$. 这些高阶方法均 可扩展至 $n$ 阶, 通常其前 $n-1$ 阶均是常规的差分 运算, 累积提取特定方向上的高阶空间信息, 第 $n$ 阶则编码不同方向上的第 $n-1$ 阶导数之间的关系. Zhang 等 ${ }^{[14]}$ 首次将局部高阶算子应用于人脸识别 研究, 提出一种局部导数模式(local derivative pattern, LDP)的人脸特征表示方法. 该方法第 $n$ 阶编 码了在局部区域某方向上中心像素与邻域像素间 的高阶导数方向变化. 与一阶导数类型的 LBP 相 比, LDP 在人脸识别中取得了更好的识别效果. LDP 码长度为 $4 \times 8$ bit, 为 LBP 的 4 倍, 特征长度的 增加也在一定程度上限制了人脸识别方法的普适 性. 为了提升人脸识别效率, Chakraborty 等 ${ }^{[15]}$ 提出 局部方向导数模式(local directional gradient pattern, LDGP), 第 $n$ 阶仅提取参考像素点在不同方 向之间的高阶导数信息，大大降低了特征的长度， 仅产生 6 bit 二进制码. 由于其仅提取参考像素这 一孤点的高阶信息, 没有关联邻域信息, 割裂了整
体与局部的关系，因此没有达到理想的识别效果. Chakraborty 等 ${ }^{[16]}$ 在此基础上提出局部梯度六元模 式(local gradient hexa pattern, LGHP), 第 $n$ 阶编码 了参考像素与邻域像素在不同距离、不同方向之间 的高阶导数关系. 高阶导数信息既存在于不同的 导数方向上, 也存在于局部邻域中. 该方法考虑各 个关系对特征表达的作用, 但是特征维数过高, 产 生较多冗余信息.

综上所述，目前基于高阶特征的人脸识别方 法还存在如下问题.

(1) 在不断追求高阶数提取图像精细鉴别信 息的同时, 并没有考虑在高阶方法中固有的噪声 敏感问题，造成方法对变化因素鲁棒性差.

(2) 在识别效率和识别率上难以平衡. 要么特 征简短, 但识别率不高, 如 LDGP; 要么识别率高, 但特征冗长, 如 LGHP.

针对现有的高阶提取方法存在的问题, 本文 提出一种基于 “梯度脸” 特征的局部高阶主方向模 式 (local high-order principal direction pattern, LHOPDP)的特征提取方法, 记为 F_LHOPDP; 另 外, 把基于 “灰度脸”的局部高阶主方向模式, 记为 G_LHOPDP. 针对第 1 个问题, 考虑在提取高阶导 数信息之前, 对灰度图像进行处理. 首先, 对灰度 图像提取多方向上的梯度分量并求和后逐点替换 原像素, 形成梯度脸结构, 该结构在底层对噪声进 行一次抑制, 提取出人脸的主要特征, 为高阶特征 提取打下基础. 针对第 2 个问题, 为了追求效率与 效果的统一性, 在最后一阶导数计算上, 设计了一 种主方向分组的编码策略, 按照 3 个主方向分组分 别编码参考像素与邻域像素的导数方向变化, 3 个 主方向分组仅产生 3 幅特征图, 且涵盖所有方向信 息, 相对于传统的 6 方向对编码策略 ${ }^{[16]}$, 特征长度 减少一半. 这样, 在保证特征丰富性的同时, 保持 了更高的识别效率.

\section{1 局部高阶导数模式的演进}

$\mathrm{LBP}^{[10]}$ 是计算机视觉中用于分类的视觉算子. 其核心思想是: 以中心像素的灰度值作为阈值, 与 其邻域像素进行大小比较得到相对应的二进制码 表示局部纹理特征. LBP 在概念上被视为无方向的 一阶局部模式算子, 其直方图特征包含了图像粗 略的边缘、斑点和其他局部特征, 特征长度为 $8 \mathrm{bit}$.

$\mathrm{LDP}^{[14]}$ 是一种基于局部导数变化的编码方向 信息的一般框架. 在该框架中, LDP 编码的是一阶 
局部模式无法从图像中获取的拥有对纹理特征精 细描述能力的高阶导数信息. LDP 算子对灰度图分 别提取 4 个方向的高阶导数信息, 并未考虑不同方 向之间存在的高阶导数信息, 得到的 LDP 码长度 为 $4 \times 8$ bit.

$\mathrm{LDGP}^{[15]}$ 同样作为高阶描述符, 考虑了 4 个方 向两两方向之间的高阶导数关系，但是忽略了邻 域像素的作用，存在提取信息不完全的问题. 但在 编码长度方面, LDGP 码长度仅为 6 bit, 甚至低于 LBP 的 8 bit.

Chakraborty 等 ${ }^{[16]}$ 在此基础上提出了 LGHP, 得到一种较为完善的高阶信息提取方案. 它依然 编码不同导数空间之间的信息, 并且引人了邻域 8 个像素点. 由于差异信息不仅存在于不同的导数 方向上，也存在于局部邻域上，因此提取的特征信 息更充分, 取得了比 LDGP 更高的识别率; 但是, LGHP 码长度为 $6 \times 9$ bit, 严重影响了识别效率. LGHP 码计算过程如下.

如图 1 所示, 在图像片段 $I(G)$ 中, $G_{0}$ 为中心 像素点, $G_{i}(i=1,2, \cdots, 8)$ 为中心像素点的邻域像素 点, 那么沿着 $\alpha=\left\{0^{\circ}, 45^{\circ}, 90^{\circ}, 135^{\circ}\right\}$ 方向的一阶导 数 $G_{\alpha}^{\prime}$ 定义为

$$
\begin{aligned}
& G_{0^{\circ}}^{\prime}\left(G_{0}\right)=G\left(G_{0}\right)-G\left(G_{4}\right) \\
& G_{45^{\circ}}^{\prime}\left(G_{0}\right)=G\left(G_{0}\right)-G\left(G_{3}\right) \\
& G_{90^{\circ}}^{\prime}\left(G_{0}\right)=G\left(G_{0}\right)-G\left(G_{2}\right) \\
& G_{135^{\circ}}^{\prime}\left(G_{0}\right)=G\left(G_{0}\right)-G\left(G_{1}\right)
\end{aligned}
$$

则在方向对 $(\alpha, \beta)$ 处, 以 $G_{0}$ 为中心点的 $3 \times 3$ 邻域 中像素的二阶方向导数定义为

$$
\begin{aligned}
& \operatorname{LGHP}_{\alpha, \beta}^{2}\left(G_{0}\right)=\left\{h\left(G_{\alpha}^{\prime}\left(G_{0}\right), G_{\beta}^{\prime}\left(G_{0}\right)\right),\right. \\
& \left.h\left(G_{\alpha}^{\prime}\left(G_{1}\right), G_{\beta}^{\prime}\left(G_{1}\right)\right), \cdots, h\left(G_{\alpha}^{\prime}\left(G_{8}\right), G_{\beta}^{\prime}\left(G_{8}\right)\right)\right\} \\
& h\left(G_{\alpha}^{\prime}(G), G_{\beta}^{\prime}(G)\right)= \begin{cases}1, & G_{\alpha}^{\prime}(G)>G_{\beta}^{\prime}(G) \\
0, & \text { 其他 }\end{cases}
\end{aligned}
$$

其中, $h(\cdot, \cdot)$ 为编码二值的编码函数, 用以编码局 部邻域像素前一阶的导数方向变化; $\alpha, \beta=$ $\left\{0^{\circ}, 45^{\circ}, 90^{\circ}, 135^{\circ}\right\}$. 最终, 将 6 个方向对的 $9 \mathrm{bit}$ 二进 制码进行级联, 得到二阶导数模式

$$
\begin{aligned}
& \operatorname{LGHP}^{2}(G)=\left\{\operatorname{LGHP}_{\alpha, \beta}^{2}(G) \mid(\alpha, \beta)=\left(0^{\circ}, 45^{\circ}\right),\right. \\
& \left(0^{\circ}, 90^{\circ}\right),\left(0^{\circ}, 135^{\circ}\right),\left(45^{\circ}, 90^{\circ}\right),\left(45^{\circ}, 135^{\circ}\right), \\
& \left.\left(90^{\circ}, 135^{\circ}\right)\right\}
\end{aligned}
$$

$$
\begin{aligned}
& \operatorname{LGHP}^{n}(G)=\left\{\operatorname{LGHP}_{\alpha, \beta}^{n}(G) \mid(\alpha, \beta)=\left(0^{\circ}, 45^{\circ}\right),\right. \\
& \left(0^{\circ}, 90^{\circ}\right),\left(0^{\circ}, 135^{\circ}\right),\left(45^{\circ}, 90^{\circ}\right),\left(45^{\circ}, 135^{\circ}\right), \\
& \left.\left(90^{\circ}, 135^{\circ}\right)\right\}
\end{aligned}
$$

\begin{tabular}{|l|l|l|}
\hline$G_{1}$ & $G_{2}$ & $G_{3}$ \\
\hline$G_{8}$ & $G_{0}$ & $G_{4}$ \\
\hline$G_{7}$ & $G_{6}$ & $G_{5}$ \\
\hline
\end{tabular}

图 1 图像 $I(G)$ 示意图

\section{2 梯度脸}

传统的高阶特征提取方法在灰度脸上提取特 征. 灰度图像中虽然包含丰富的纹理信息, 但局部 区域受到光照和噪声等因素的干扰后, 经后续的 高阶特征提取放大, 会导致一些重要的细节信息 无法凸显甚至被掩盖掉，使算法性能下降. 提取梯 度特征考虑的是像素点的差异, 并依据梯度变化 程度有选择性地记录局部差异信息. 在一幅图像 中, 人们往往关注局部灰度变化剧烈的区域, 如孤 立点、线、边缘等图像特征所在的区域. 人脸图像 中的关键特征部位(如五官等梯度变化较大的区 域)分布着大量这样的图像特征. 本节通过提取多 方向的像素梯度分量再求和的方法, 提取灰度图 像梯度域信息, 构造梯度脸. 因为参考点与邻域各 点像素之间存在差异, 即存在高、低频信号, 在使 用梯度分量记录这种差异时会引人被放大的噪声 信息. 所以，考虑通过求和运算对噪声信息在低阶 底层进行一次平滑抑制，可在局部区域内一定程 度上忽略或减少噪声。人脸图像经过梯度脸算法 处理后, 不但削弱了局部区域光照变化因素的影 响, 保留了主要的人脸特征, 而且由于在底层抑制 了噪声，因此后续高阶计算中噪声的传递被降低.

如图 1 所示, 在以 $G_{0}$ 为中心点的 $3 \times 3$ 图像区 域中, 邻域 8 个像素点代表的是与中心点相差 1 或 $\sqrt{2}$ 距离的像素点. 图像中心点 $G_{0}$ 的水平、垂直和 对角方向的梯度分量表示为

$$
G_{\theta}^{\prime}\left(G_{0}\right)=G\left(G_{0}\right)-G\left(G_{i}\right)
$$

其中, $\theta=\left\{0^{\circ}, 45^{\circ}, 90^{\circ}, 135^{\circ}\right\} ; i=\{4,3,2,1\}$.

那么, 中心像素点 $G_{0}$ 的梯度幅值定义为

$$
G_{0}^{\prime}=\sum_{\theta}\left|G_{\theta}^{\prime}\left(G_{0}\right)\right|, \theta=\left\{0^{\circ}, 45^{\circ}, 90^{\circ}, 135^{\circ}\right\}
$$


通过卷积核 $\left[\begin{array}{rrr}1 & 1 & -1 \\ 1 & 0 & -1 \\ 1 & -1 & -1\end{array}\right]$ 的卷积运算取绝对值, 然后把结果相加到一起, 最终得到的值作为该像 素的新值, 扩展至整幅人脸矩阵, 以形成对应的梯 度脸，构造过程示意图如图 2 所示.

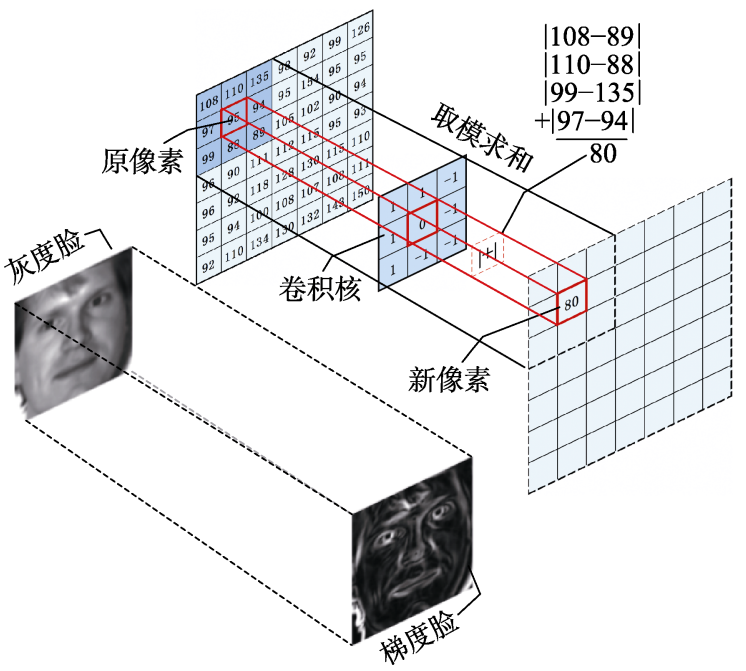

图 2 构造梯度脸示意图

\section{LHOPDP}

\section{1 主方向分组策略}

描述符对特征表达的准确性取决于如何将图 像局部邻域关系精确地映射到微观结构中. 在第 $n$ 阶导数的计算上, $\mathrm{LDGP}^{[15]}$ 和 $\mathrm{LGHP}^{[16]}$ 等方法重 点关注像素点两两方向之间的导数方向变化. 虽 然 LGHP 提取的空间信息较为充分，但是对 4 个方 向采用相等的权重分配策略, 随机组合得到 6 个方 向对, 即 6 个方向模板独立产生 6 幅特征图, 再进 行级联, 导致了特征长度的增加, 造成人脸识别时 间上的延长. 为了平衡识别效率与识别率之间的 关系，本节依然抓住局部区域像素在不同方向之 间的高阶导数关系的构建, 采取了更为精简的编 码方式, 引人了主方向分组策略. 为了突出某一方 向的作用，设定主方向并以此为参考，将与主方向 相关的方向尽可能多地划分到对应的分组中，即 (1) $0^{\circ}$ 主方向: $\left(0^{\circ} \mid 45^{\circ}, 90^{\circ}, 135^{\circ}\right)$; (2) $45^{\circ}$ 主方向: $\left(45^{\circ} \mid 90^{\circ}, 135^{\circ}\right)$; (3) $90^{\circ}$ 主方向: $\left(90^{\circ}, 135^{\circ}\right)$. 不同 于 LGHP 编码图像邻域内单一方向对的导数方向变 化, 这里编码的是图像邻域内主方向的导数方向 变化，使分组中涵盖了更多的方向信息. 在最后一 阶上统计分组中与主方向同向邻域方向的个数,
同向的越多, 视为对主方向的加强, 予以保留. 这 样，多个不同方向对的导数空间信息就集中分布 于主方向组中，消除了过多的冗余信息，进而减少 了特征长度, 特征长度降低为 $3 \times 9$ bit, 仅为 LGHP 方法的一半, 有效地提升了识别效率. 同时, 第 $n$ 阶仅保留粗糙的导数方向转换信息, 而不是像前 $n-1$ 阶那样使用传统的差分信息, 以降低在高阶 空间提取信息的噪声敏感性. 主方向分组策略示 意过程如图 3 所示.

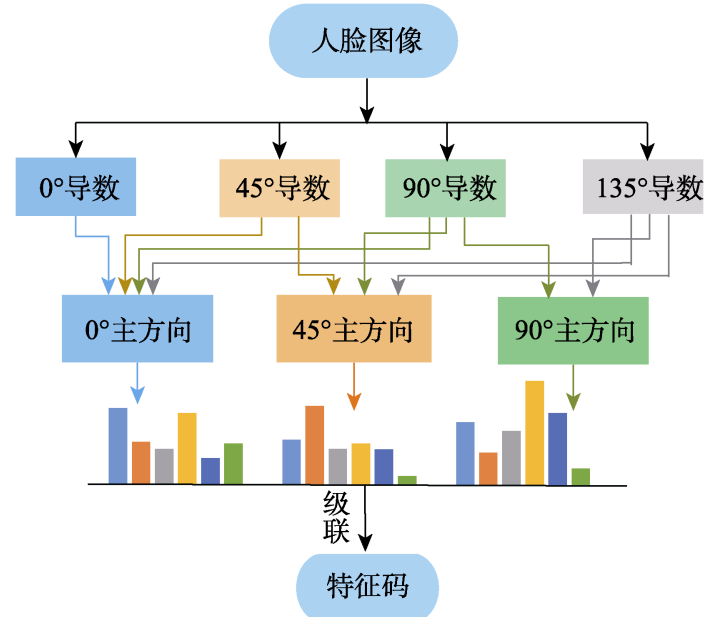

图 3 主方向分组策略示意图

\section{2 特征编码}

以二阶 LHOPDP 的特征提取方法为例说明具 体特征编码过程. 4 个方向的一阶导数计算方法同 式(1) 式(4). 对于二阶导数, 如图 1 中的局部图像 $I(G)$ 所示, 从 $0^{\circ}$ 方向开始沿逆时针方向, 分别以 $0^{\circ}, 45^{\circ}$ 和 $90^{\circ}$ 为主方向, 在 $3 \times 3$ 邻域内, 计算组内主 方向与邻域方向之间的导数, 按照规则进行编码.

(1) $0^{\circ}$ 主方向: $\left(0^{\circ} \mid 45^{\circ}, 90^{\circ}, 135^{\circ}\right)$

以 $0^{\circ}$ 作为主方向的二阶方向导数定义为

$$
\begin{aligned}
& \text { LHOPDP } P_{\theta \mid \alpha, \beta, \gamma}^{2}(G)= \\
&\left\{P\left(C_{\theta, \alpha}^{2}\left(G_{0}\right), C_{\theta, \beta}^{2}\left(G_{0}\right), C_{\theta, \gamma}^{2}\left(G_{8}\right), C_{\theta, \beta}^{2}\left(G_{0}\right), C_{\theta, \gamma}^{2}\left(G_{8}\right)\right)\right\}, \\
& C_{\theta, \phi}^{2}(G)=G_{\theta}^{\prime}(G)-G_{\phi}^{\prime}(G)=\left\{\begin{array}{l}
1, G_{\theta}^{\prime}(G)>G_{\phi}^{\prime}(G) \\
0, \text { 其他 }
\end{array}\right. \\
& P\left(C_{\theta, \alpha}^{2}(G), C_{\theta, \beta}^{2}(G), C_{\theta, \gamma}^{2}(G)\right)= \\
&\left\{\begin{array}{l}
1, C_{\theta, \alpha}^{2}(G)+C_{\theta, \beta}^{2}(G)+C_{\theta, \gamma}^{2}(G) \geqslant 2 \\
0, \text { 其他 }
\end{array}\right.
\end{aligned}
$$


其中, $\theta$ 为主方向 $0^{\circ} ; \alpha, \beta$ 和 $\gamma$ 是主方向的邻域 方向, 取值分别为 $45^{\circ}, 90^{\circ}$ 和 $135^{\circ} ; C_{\theta, \phi}^{2}(G)$ 为符 号函数，用以记录主方向的导数变化; $P(\cdot,,$,$) 为$ 编码函数. 主方向与邻域方向的导数方向变化关 系共有 4 种情况: “111” “110” “100”和“000”. 例如， “111”表示的是有 3 个邻域方向与主方向在导数方 向上同向. 与主导数方向同向的邻域方向越多, 视 为对主方向的加强, 这里设置阈值为 2 , 表示在组 内存在 2 个及以上与主方向导数同向的情况编码 为“ 1 ”, 反之编码为“ 0 ”.

(2) $45^{\circ}$ 主方向: $\left(45^{\circ} \mid 90^{\circ}, 135^{\circ}\right)$

同理, $45^{\circ}$ 主方向的二阶方向导数定义为

$$
\begin{aligned}
& \operatorname{LHOPDP}_{\theta \mid \beta, \gamma}^{2}(G)=\left\{P\left(C_{\theta, \beta}^{2}\left(G_{0}\right), C_{\theta, \gamma}^{2}\left(G_{0}\right)\right),\right. \\
& \left.\quad \cdots, P\left(C_{\theta, \beta}^{2}\left(G_{8}\right), C_{\theta, \gamma}^{2}\left(G_{8}\right)\right)\right\} \\
& P\left(C_{\theta, \beta}^{2}(G), C_{\theta, \gamma}^{2}(G)\right)= \\
& \left\{\begin{array}{l}
1, C_{\theta, \beta}^{2}(G)+C_{\theta, \gamma}^{2}(G) \geqslant 2 \\
0, \text { 其他 }
\end{array}\right.
\end{aligned}
$$

其中, $\theta$ 为主方向 $45^{\circ} ; \beta$ 和 $\gamma$ 为沿逆时针方向的 2 个邻域方向, 取值分别为 $90^{\circ}$ 和 $135^{\circ}$. 这里导数方 向变化有 “ 11 ”“ 10 ”和 “ 00 ” 3 种情况. 编码函数 $P(\cdot, \cdot)$ 的阈值同样设置为 2 .

(3) $90^{\circ}$ 主方向: $\left(90^{\circ}, 135^{\circ}\right)$

$$
\begin{array}{|l|l|l|}
\hline G_{1} & G_{2} & G_{3} \\
\hline G_{8} & G_{0} & G_{4} \\
\hline G_{7} & G_{6} & G_{5} \\
\hline
\end{array}
$$

a. 编码模板
以 $90^{\circ}$ 为主方向的二阶方向导数定义为

$$
\begin{aligned}
& \operatorname{LHOPDP}_{\theta \mid \gamma}^{2}(G)=\left\{P\left(C_{\theta, \gamma}^{2}\left(G_{0}\right)\right), \cdots,\right. \\
& \left.P\left(C_{\theta, \gamma}^{2}\left(G_{8}\right)\right)\right\} \\
& P\left(C_{\theta, \gamma}^{2}(G)\right)= \begin{cases}1, & C_{\theta, \gamma}^{2}(G)=1 \\
0, & \text { 其他 }\end{cases}
\end{aligned}
$$

其中， $\theta$ 为主方向 $90^{\circ} ; \gamma$ 为沿着逆时针方向的一 个邻域方向 $135^{\circ}$. 这里导数方向变化有“1”和“ 0 ” 2 种情况，编码函数 $P(\cdot)$ 的阈值设置为 1.

最终, 级联 3 个主方向的二进制码, 得到二阶 局部主方向模式为

$$
\begin{gathered}
\operatorname{LHOPDP}^{2}(G)=\left\{\operatorname{LHOPDP}_{0^{\circ} \mid 45^{\circ}, 90^{\circ}, 135^{\circ},}^{2},\right. \\
\left.\operatorname{LHOPDP}_{45^{\circ} \mid 90^{\circ}, 135^{\circ}}^{2}, \operatorname{LHOPDP}_{90^{\circ} \mid 135^{\circ}}^{2}\right\}
\end{gathered}
$$

扩展至 $n$ 阶为

$$
\begin{aligned}
& \operatorname{LHOPDP}^{n}(G)=\left\{\operatorname{LHOPDP}_{0^{\circ} / 45^{\circ}, 90^{\circ}, 135^{\circ}}^{n},\right.
\end{aligned}
$$

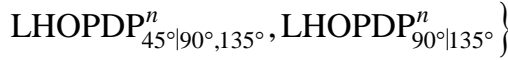

图 4 所示为 $0^{\circ}$ 主方向的二阶 LHOPDP 编码过 程. 图 4a 指示了编码的开始方向, 即沿着逆时针 取 4 个方向依次求导数. 图 4c 中计算出方向导数 并将 3 个导数方向变化二值化, 根据编码策略提取 特征码. 同理, 可以分别计算出 $45^{\circ}$ 和 $90^{\circ}$ 主方向 的特征码, 最终级联形成 LHOPDP 码.

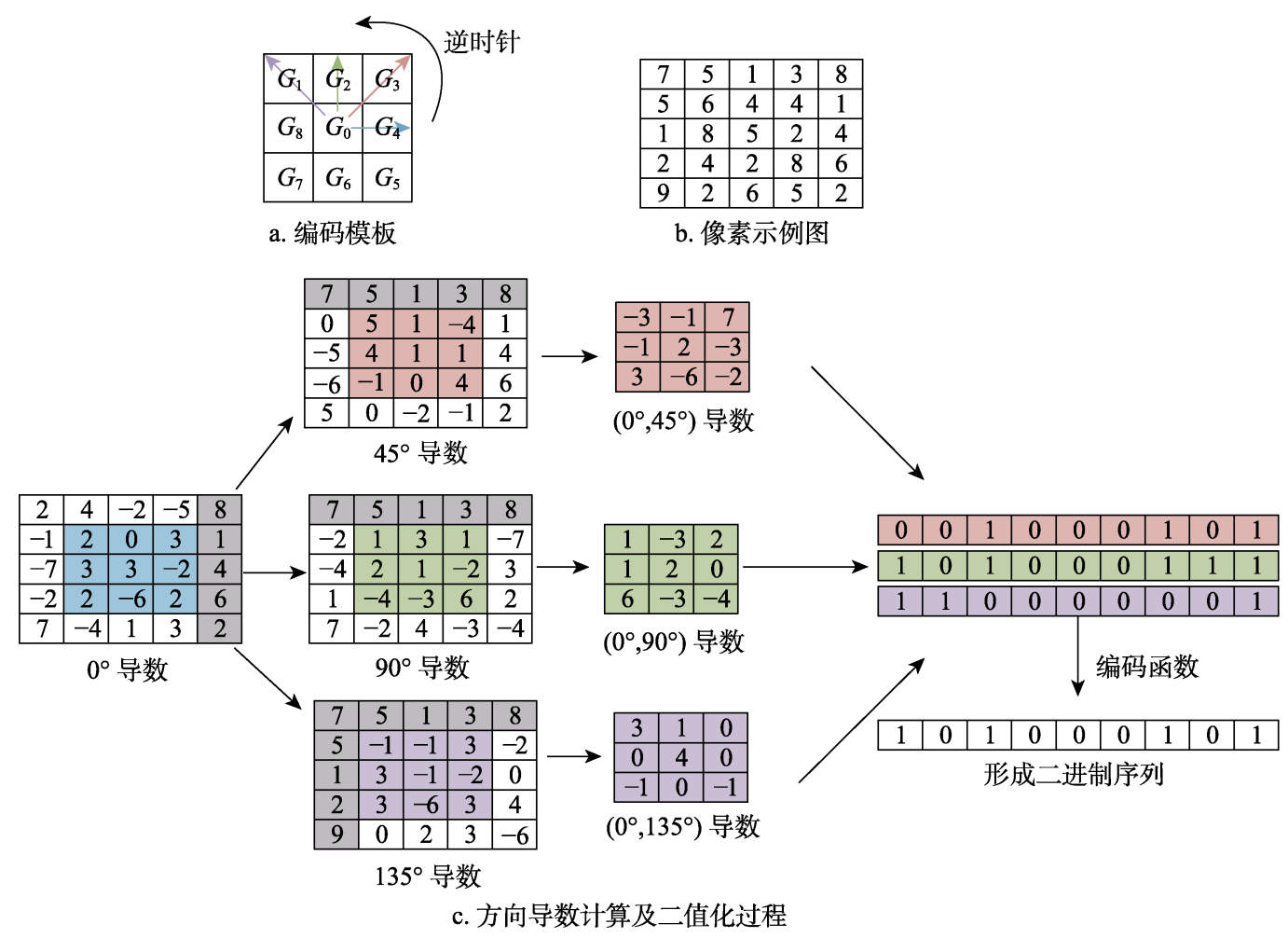

图 $40^{\circ}$ 主方向的二阶 LHOPDP 编码过程 


\section{3 纹理特征图的对比与分析}

人脸纹理特征的表达效果与最终的识别效果 有较好的一致性. 因此, 可通过人脸的纹理特征图 比较直观地观察、对比不同基于局部特征的人脸识 别方法. 为了更直观地了解不同特征提取方法的 差异性，本文选取无变化因素和存在表情、遮挡、 光照等变化因素的人脸图像, 分别用 $\mathrm{LBP}^{[10]}$, $\mathrm{LDP}^{[14]}, \mathrm{LDGP}^{[15]}, \mathrm{LGHP}^{[16]}$ 和本文的 G_LHOPDP 和 F_LHOPDP 方法对上述图像进行纹理提取. 其中, 对比方法 G_LHOPDP 表示在灰度脸上提取特征, 简记为 G_1; F_LHOPDP 表示在第 2 节所提出的梯 度脸上提取特征，简记为 F_2. 每种高阶方法均取 一幅 3 阶特征图以示说明, 如图 5 所示.

从图 5 可以看出, 在灰度变化较大区域, 如轮 廓、眼睛、鼻子和嘴巴等分布的地方，作为图像的一
阶导数模式, LBP 提取纹理会产生较粗的边缘, 特征 呈块状, 对图像的细节描述能力不够; LDP 在提取某 一方向的高阶导数信息时, 未考虑其他相邻方向对 此方向的影响, 忽略了相邻方向之间存在的高阶导 数信息, 导致轮廓刻画能力不够. LDGP 虽然注意到 不同方向导数之间关系的构建, 但其仅提取参考像 素点的高阶信息, 并没有关联到邻域信息, 割裂了 整体与局部的关系, 纹理细节不够丰富. LGHP 和 G_1 不仅在前 $n-1$ 阶累积提取到了同一方向上的高 阶空间信息，也在第 $n$ 阶捕捉到了不同方向之间的 高阶空间信息，同时又补充了邻域信息，提取了更 为详尽的纹理信息. 高阶方法提取纹理固然较为细 淢, 尤其在细节处理方面, 但是随着阶数的提高, 特 征信息中将引入越来越多的噪声，提取阶数达到 4 阶后特征图将被噪声淹没, 难以识别 ${ }^{[14-15]}$.

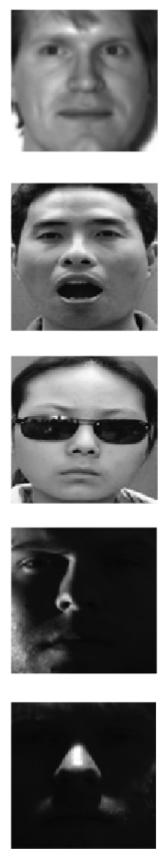

a. 原图
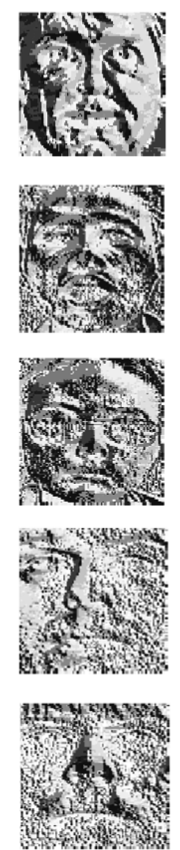

b. $\mathrm{LBP}^{[10]}$
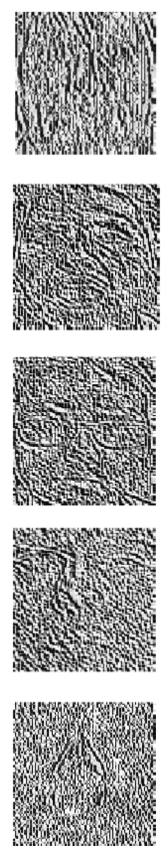

c. $\operatorname{LDP}^{[14]}$
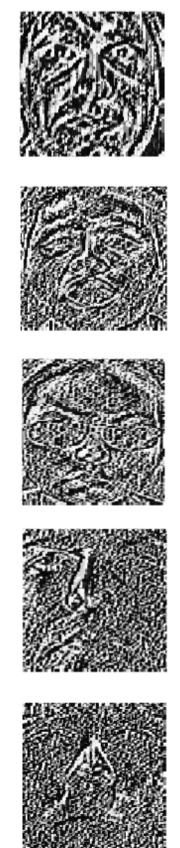

d. $\operatorname{LDGP}^{[15}$
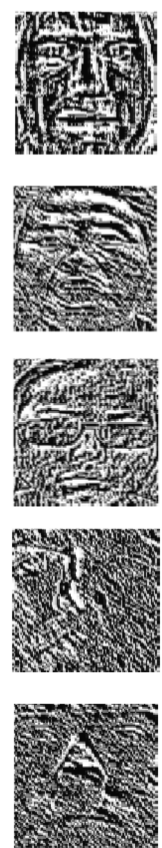

e. $\mathrm{LGHP}^{[16]}$
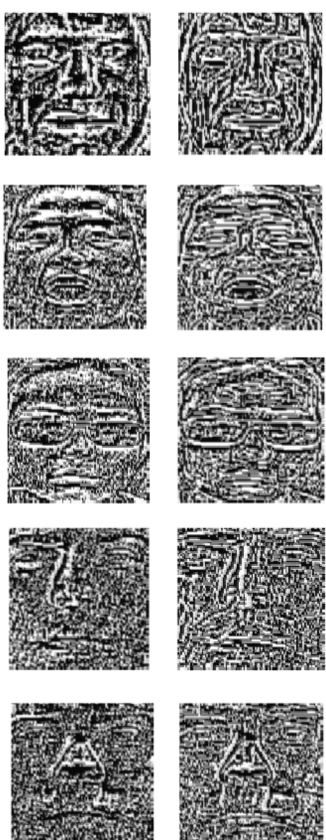

f. $G \_1$

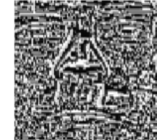

g. F_2

图 56 种方法纹理图对比

F_2 在特征域上即梯度脸上，采用了主方向分 组策略的高阶空间信息提取方式. 可以看出，在与 同类方法保持相同提取阶数的条件下，同类方法 出现了不同程度的噪声掩盖纹理特征的情况, 模 糊不清. 该方法提取的纹理图黑色斑块减少, 纹理 线条更加细㖑突出, 尤其对严重光照的人脸, 也能 有较好的刻画能力. 该方法在一定程度上减弱了 高阶方法放大噪声对提取特征带来的影响.

\section{4 方法步骤}

本文方法步骤如下.

Step1. 对原始人脸灰度图像进行梯度脸特征初提取.
Step2. 分块处理梯度脸图像, 利用 LHOPDP 对梯 度脸特征进行高阶空间特征提取, 获得特征图像.

Step3. 统计获得每块特征图像的直方图特征并进 行级联，作为该幅人脸图像的识别特征.

Step4. 最后利用多分类支持向量机(support vector machine, SVM)进行匹配识别, 计算出识别率并统计识 别时间.

\section{4 实验结果与分析}

\section{1 仿真环境和参数设置}

为了验证本文方法性能, 实验数据选择了包含 
光照、表情、遮挡和姿态等变化因素的公开人脸库, 与几种前沿的同类方法进行仿真实验. 这些方法包 括 $\mathrm{LBP}^{[10]}$, $\mathrm{LDP}^{[14]}$, $\mathrm{LDGP}^{[15]}$ 和 $\mathrm{LGHP}^{[16]}$. 为保证实验 的公平性, 所有方法均采用最优分块, 分块采样步 长均为 1 , 提取阶数均为 3 阶, 并且 CASE-PEAL 库 和 Extended Yale B 库图像大小归一到 $100 \times 100$. CMU PIE 库样本量庞大, 为了减少识别时间, 图像 大小归一化到 $64 \times 64$. 直方图的 bin 数统一设置为 20. CASE-PEAL 库用于单独测试方法对单一变化 因素的鲁棒性，由于其光照集、表情集和饰物集的 类数以及每类中的人脸数目各不相同, 仅设定训 练、测试样本比例分别为 $4: 5,2: 3$ 和 $3: 3$, 用以 测试各类算法. Extended Yale B 库和 CMU PIE 库 分别随机选取每类样本的 $10 \%$ 40\%作为训练样本, 对应剩余比例的样本作为测试样本, 随机测试 10 次 取平均识别率作为最终识别率. 仿真环境为 Windows7 操作系统, AMD Phenom ${ }^{(\mathrm{TM})}$ II X4 9553.2GHz CPU, 12 GB RAM, Matlab R2018a. 鉴 于 SVM 在小样本、非线性样本条件下良好的效果 和效率 ${ }^{[19]}$, 分类器选择 SVM, 并且搭配适用于多分 类问题的 libSVM 软件包 ${ }^{[20]}$.

\subsection{CASE-PEAL 数据库的结果与分析}

CASE-PEAL 库是大规模中国人脸数据库, 共 包含 1040 名中国人的共 99450 幅人脸图像，该人 脸库涵盖了光照、表情、饰物和姿态 4 种主要变化 条件. 其中, CASE-PEAL 光照集共 232 人, 每人 9 幅人脸图像; 表情集共 376 人，每人 5 幅人脸图像; 饰物集共 433 人, 每人 6 幅人脸图像. 饰物集饰物 的变化种类有 6 种，包括各款式眼镜、黑色墨镜、 帽子等饰品, 用于测试遮挡变化因素. 部分样本如 图 6 所示.

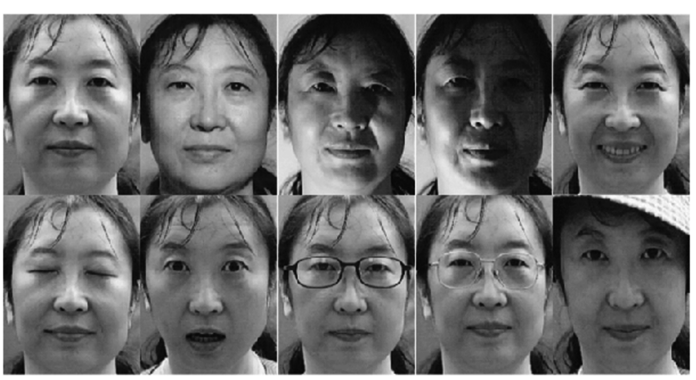

图 6 CASE-PEAL 库部分样本

6 种方法在 CASE-PEAL 库的识别率结果如表 1 所示. 由于 CASE-PEAL 库每个子集人数均不同, 本次实验选择样本数最多的饰物集进行时间统计 实验, 以示说明. 图 7 记录了 5 种高阶方法在
CASE-PEAL 库饰物集的单次识别时间，其中，识 别总时间为特征提取时间与特征匹配时间之和.

表 16 种方法在 CASE-PEAL 库的识别率 \%

\begin{tabular}{lccc}
\hline \multicolumn{1}{c}{ 方法 } & 光照集 & 表情集 & 饰物集 \\
\hline LBP $^{[10]}$ & 75.16 & 98.42 & 96.65 \\
LDP $^{[14]}$ & 90.22 & 98.62 & 94.23 \\
LDGP $^{[15]}$ & 85.19 & 98.71 & 93.18 \\
LGHP $^{[16]}$ & 89.36 & 99.14 & 95.91 \\
G_LHOPDP & 88.93 & 99.15 & 96.42 \\
F_LHOPDP & 93.48 & 99.13 & 97.42 \\
\hline
\end{tabular}

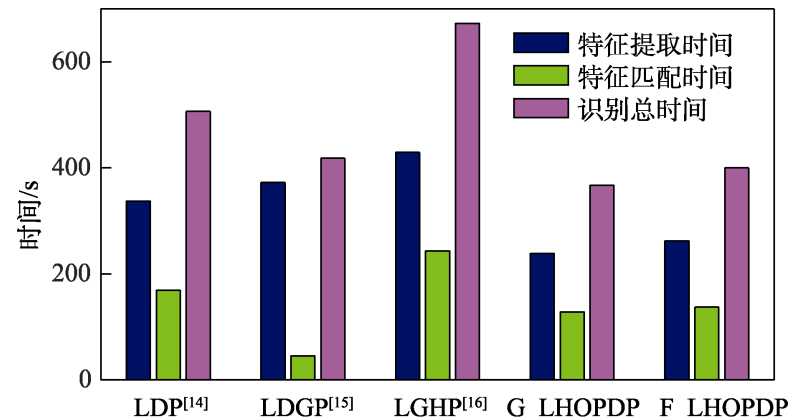

图 75 种方法在 CASE-PEAL 饰物集时间消耗比较

从表 1 可以看出，本文方法的识别率均不同程 度优于同类方法. 在光照集下, 作为一阶模式, LBP 特征按照邻域像素的灰度关系进行编码, 鲁 棒性较差, 对于随机噪声、光照变化等外界环境变 化比较敏感. 而高阶方法编码不同导数空间的方 向信息, 抵抗光照变化能力增强. 对于表情变化, 各种方法均能提取详尽的鉴别信息, 识别率较高, F_LHOPDP 的识别率达到 99.13\%. 在饰物集中, LDP 仅考虑高阶空间单一方向的导数变化, 识别 率为 $94.23 \%$; LDGP 虽然考虑了不同方向导数之间 存在的空间信息, 但是忽略了像素的邻域信息, 识 别率为 93.18\%; LGHP 汲取 LDP 和 LDGP 的优点, 提取了较为丰富的特征信息, 识别率达到了 $95.91 \%$.

值得说明的是, 出于严谨性的考虑, 实验存在 一个从 G_LHOPDP 到 F_LHOPDP 演进过程. F_LHOPDP 的初级阶段 G_LHOPDP 是利用主方向 分组策略的编码方式对灰度图直接提取高阶特征, 目的是在准确表达高阶空间中各种关系的基础上, 调整编码方式，进而降低特征的长度，提升识别效 率. 编码模板从传统的方向对向方向组的转变, 使 每个方向组内涵盖更多的高阶导数信息, 但是因 为仅仅是以主方向作为参考，不同方向之间的高 阶导数信息只能粗略地分布于 3 个方向组内, 导致 
特征级联后长度直接减半, 从实验的结果来看, 该 方法的效果与 LGHP 相当, 多数情况下并未优于 LGHP, 而F_LHOPDP 选择在梯度脸上利用主方向 分组策略提取高阶特征, 提高了抗噪性能, 在光照 集、饰物集中平均识别率比 LGHP 方法分别提高 了 $4.12 \%$ 和 $1.51 \%$.

特征提取时间是衡量算法复杂度的一个重要 指标. 如图 7 所示, F_LHOPDP 的特征提取时间均 低于 LDP, LDGP 和 LGHP. 特征匹配时间主要取决 于特征的维数, F_LHOPDP 的特征匹配时间仅高于 LDGP，但是综合起来看，对于单次识别总时间， F_LHOPDP 均低于其余方法, 分别比 LDP, LDGP 和 LGHP 低了 $106.4 \mathrm{~s}, 18.0 \mathrm{~s}$ 和 $272.3 \mathrm{~s}$.

\subsection{Extended Yale B 数据库的结果与分析}

Extended Yale B 是一个专门用来测试算法对 光照变化鲁棒性的基准数据库, 包含 38 个人的 64 种不同光照变化的图像. 部分样本如图 8 所示. 6 种方法在 Entended Yale B 库的识别率结果如表 2 所示，高阶方法的时间度量结果如图 9 所示.

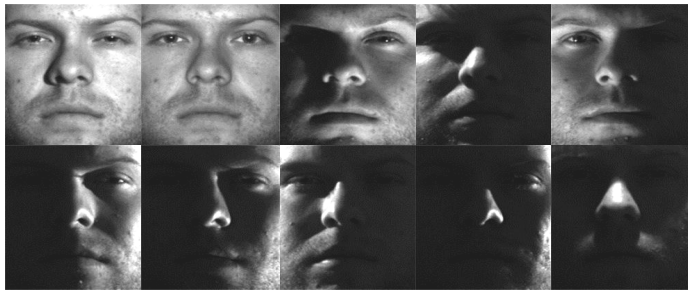

图 8 Extended Yale B 库部分样本

表 26 种方法在 Extended Yale B 库的识别率 \%

\begin{tabular}{lcccc}
\hline \multirow{2}{*}{ 方法 } & \multicolumn{4}{c}{ 每类随机选择样本比例 } \\
\cline { 2 - 5 } & $10 \%$ & $20 \%$ & $30 \%$ & $40 \%$ \\
\hline LBP $^{[10]}$ & 70.53 & 73.73 & 93.73 & 98.18 \\
LDP $^{[14]}$ & 79.04 & 91.90 & 96.98 & 98.12 \\
LDGP $^{[15]}$ & 80.55 & 93.82 & 97.94 & 98.68 \\
LGHP $^{[16]}$ & 85.49 & 96.26 & 98.43 & 98.64 \\
G_LHOPDP & 84.54 & 95.65 & 98.33 & 99.38 \\
F_LHOPDP & 91.81 & 98.79 & 99.65 & 99.85 \\
\hline
\end{tabular}

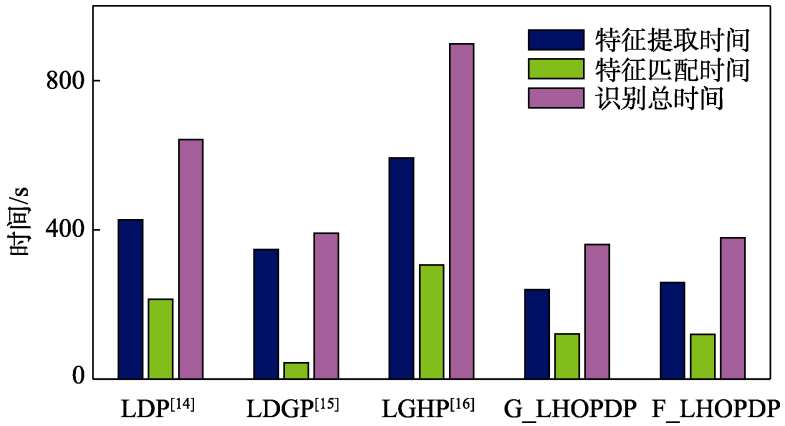

图 95 种方法在 Extended Yale B 库时间消耗比较
从表 2 可以看出, 在强烈光照因素的影响下, 高阶导数方法整体识别率要高于 LBP, 高阶方法提 取更稳健的方向信息，提高了对光照变化的鲁棒 性。在 $10 \%$ 的训练样本比例下, LGHP 和 G_LHOPDP 识别率相当, F_LHOPDP 在对局部光 照变化更鲁棒的梯度特征上提取高阶信息, 相比 其他方法表现更佳, 识别率分别比 LBP, LDP, LDGP, LGHP 和 G_LHOPDP 提高了 $21.28 \% ， 12.77 \%$, $11.26 \%$ ，6.32\%和 $7.27 \%$, 对小样本的表现更突出. 结合图 9, 在识别效率方面, F_LHOPDP 因其不高 的特征维数带来了时间上的优势，特征提取时间 相比其他方法最低，特征匹配时间仅高于 LDGP, 综合来看, 识别总时间与特征码仅有 6bit 的 LDGP 码相当, 但是识别率要提高 $11.26 \%$.

\subsection{CMU PIE 数据库的结果与分析}

CMU PIE 人脸库共采集 68 人的人脸数据, 总 样本数达到 41368 幅, 其中, 每类样本包括 13 种 姿态变化、43 种光照变化和 4 种表情变化, 部分样 本如图 10 所示, 6 种方法在 CMU PIE 库的识别率 结果如表 3 所示. 图 11 所示为 5 种高阶方法的时 间度量结果.

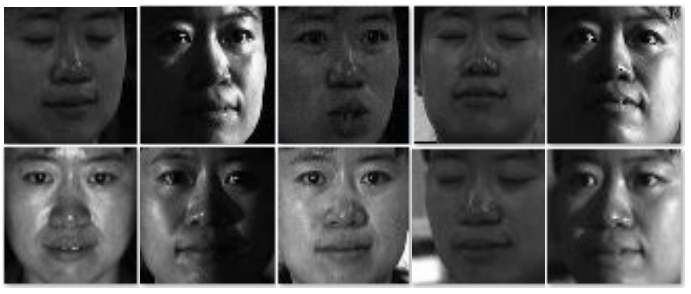

图 10 CMU PIE 库部分样本

表 36 种方法在 CMU PIE 库的识别率 \%

\begin{tabular}{lcccc}
\hline \multirow{2}{*}{ 方法 } & \multicolumn{4}{c}{ 每类随机选择样本比例 } \\
\cline { 2 - 5 } & $10 \%$ & $20 \%$ & $30 \%$ & $40 \%$ \\
\hline LBP $^{[10]}$ & 60.32 & 63.99 & 69.52 & 71.98 \\
LDP $^{[14]}$ & 71.55 & 75.99 & 81.73 & 85.00 \\
LDGP $^{[15]}$ & 69.12 & 73.30 & 78.32 & 81.45 \\
LGHP $^{[16]}$ & 82.85 & 86.52 & 90.69 & 92.65 \\
G_LHOPDP & 81.20 & 86.65 & 89.77 & 91.69 \\
F_LHOPDP & 85.33 & 88.20 & 91.17 & 93.01 \\
\hline
\end{tabular}

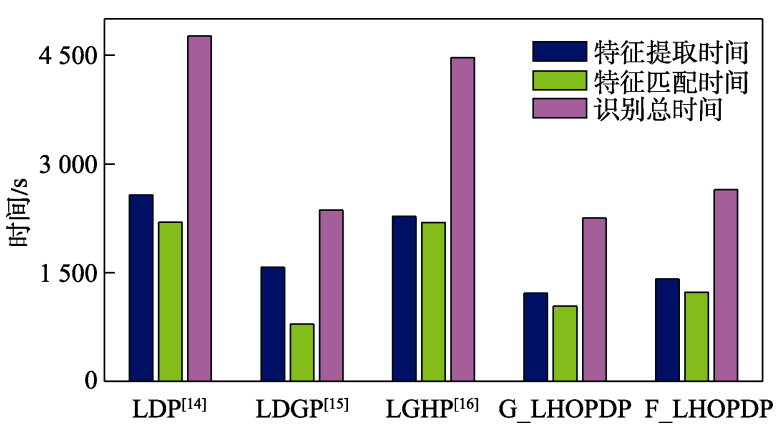

图 115 种方法在 CMU PIE 库时间消耗比较 
从表 3 可以看出, 在姿态和光照双重因素的影 响下, 与对比方法中性能最优的 LGHP 相比, 在选择 10\%训练样本的情况下, 本文方法 F_LHOPDP 识别 率仍高出 $2.48 \%$. 从图 11 可以看出, F_LHOPDP 的 识别总时间与特征长度仅有 $6 \mathrm{bit}$ 的 LDGP 相当, 但 是比最近主流方法 LGHP 低了 $1824.3 \mathrm{~s}$. 对于 CMU PIE 这样的大型数据库, 特征长度对匹配时间的影 响较大, 进而影响识别总时间, 可以看出, 随着样 本量的增加, 提取低特征维数的方法在人脸识别 系统中占据优势. 本文方法在保证识别率的前提 下将时间复杂度降低至一个可接受的水平.

\section{5 结 语}

针对现有高阶特征的人脸识别因对噪声敏感 导致的鲁棒性差以及特征冗长的问题, 本文提出了 基于梯度脸的局部高阶主方向模式(F_LHOPDP)的 人脸识别方法. 通过在 CASE-PEAL, Extended Yale B 和 CMU PIE 人脸库的实验表明, 本文方法相比 其他方法对人脸光照变化、表情变化和面部遮挡变 化因素具有更优良的鲁棒性，较好地缓解了高阶 方法噪声敏感问题，且在识别率和识别效率上达 到了一个较好的平衡.

未来将考虑结合人脸的多维特征(多尺度、多 方向等)与高阶特征的表达进行研究，进一步提高 人脸特征的表达能力.

\section{参考文献(References):}

[1] Liu L, Fieguth P, Guo Y L, et al. Local binary features for texture classification: Taxonomy and experimental study[J]. Pattern Recognition, 2017, 62: 135-160

[2] Chakraborty S, Singh S K, Chakraborty P. Cascaded asymmetric local pattern: a novel descriptor for unconstrained facial image recognition and retrieval[J]. Multimedia Tools and Applications, 2019, 78(17): 25143-25162

[3] Cevik N, Cevik T. DLGBD: a directional local gradient based descriptor for face recognition[J]. Multimedia Tools and Applications, 2019, 78(12): 15909-15928

[4] Kim C, Klabjan D. A simple and fast algorithm for L1-norm kernel PCA[J]. IEEE Transactions on Pattern Analysis and Machine Intelligence, 2020, 42(8): 1842-1855

[5] Ye Q L, Zhao H H, Fu L Y. Underlying connections between algorithms for nongreedy LDA-L1[J]. IEEE Transactions on Image Processing, 2018, 27(5): 2557-2559

[6] Xu Jiayi, Xue Xinying, Li Jianjun, et al. Composite sketch recognition using multi-scale HOG features and semantic attributes[J]. Journal of Computer-Aided Design \& Computer Graphics, 2020, 32(2): 297-304(in Chinese)

(许佳奕, 薛釒营, 李建军, 等. 结合多尺度 HOG 特征和语 义属性的合成素描人脸识别 [J]. 计算机辅助设计与图形学 学报, 2020, 32(2): 297-304)

[7] Li C R, Huang Y Y, Xue Y. Dependence structure of Gabor wavelets based on copula for face recognition[J]. Expert Systems with Applications, 2019, 137: 453-470

[8] Tan X Y, Triggs B. Enhanced local texture feature sets for face recognition under difficult lighting conditions[J]. IEEE Transactions on Image Processing, 2010, 19(6): 1635-1650

[9] Yang S F, Bhanu B. Understanding discrete facial expressions in video using an emotion avatar image[J]. IEEE Transactions on Systems Man and Cybernetics, Part B: Cybernetics, 2012, 42(4): 980-992

[10] Ojala T, Pietikainen M, Maenpaa T. Multiresolution gray-scale and rotation invariant texture classification with local binary patterns[J]. IEEE Transactions on Pattern Analysis and Machine Intelligence, 2002, 24(7): 971-987

[11] Liu J, Chen Y, Sun S N. A novel local texture feature extraction method called multi-direction local binary pattern[J]. Multimedia Tools and Applications, 2019, 78(13): 18735-18750

[12] Sun K, Yin X, Yang M X, et al. The face recognition method based on CS-LBP and DBN[J]. Mathematical Problems in Engineering, 2018, 2018: Article No.3620491

[13] Lee K, Jeong T, Woo S, et al. Octagonal prism LBP representation for face recognition[J]. Multimedia Tools and Applications, 2018, 77(16): 21751-21770

[14] Zhang B C, Gao Y S, Zhao S Q, et al. Local derivative pattern versus local binary pattern: face recognition with high-order local pattern descriptor[J]. IEEE Transactions on Image Processing, 2010, 19(2): 533-544

[15] Chakraborty S, Singh S K, Chakraborty P. Local directional gradient pattern: a local descriptor for face recognition[J]. Multimedia Tools and Applications, 2017, 76(1): 1201-1216

[16] Chakraborty S, Singh S K, Chakraborty P. Local gradient hexa pattern: a descriptor for face recognition and retrieval[J]. IEEE Transactions on Circuits and Systems for Video Technology, 2018, 28(1): 171-180

[17] Zhu Z Q, Zheng M G, Qi G Q, et al. A phase congruency and local Laplacian energy based multi-modality medical image fusion method in NSCT domain[J]. IEEE Access, 2019, 7: 20811-20824

[18] Wang F J, Chen W, Qiu L. Hausdorff derivative Laplacian operator for image sharpening[J]. Fractals, 2019, 27(3): Article No.1950060

[19] Yao Liping, Pan Zhongliang. Research on a face recognition method based on multi-algorithms fusion[J]. Journal of Optoelectronics·Laser, 2019, 30(9): 960-967(in Chinese) (姚立平, 潘中良. 一种多算法融合的人脸识别方法研究 $[\mathrm{J}]$. 光电子·激光, 2019, 30(9): 960-967)

[20] Chang C C, Lin C J. LIBSVM: a library for support vector machines[J]. ACM Transactions on Intelligent Systems and Technology, 2011, 2(3): Article No.27 